\title{
ITERATES OF VOLTERRA OPERATORS AND INDETERMINATE FORMS
}

\author{
PABLO BRAZ E SILVA AND ANDRÉS R. R. PAPA
}

Received 10 February 2005; Revised 6 January 2006; Accepted 22 January 2006

We state and prove a theorem showing how iterates of the Volterra operator can be used to evaluate indeterminate forms of type $0 / 0$. This general result allows one to recover the Boltzmann-Gibbs entropy as a limit of a wide class of generalized entropies, as considered in an earlier work.

Copyright (c) 2006 Hindawi Publishing Corporation. All rights reserved.

\section{Introduction}

During the last decades, many attempts have been made in order to explain and formalize mathematically a large set of phenomena that, at a first glance, are not well described by traditional theories. Among those attempts one finds the whole theory of self-organized criticality and some statistical theories. In recent years, a popular approach has been a Daróczy-like entropy [2] and its corresponding statistical mechanics formalism known as Tsallis thermostatistics [12]

$$
S_{q}=k_{B} \frac{1-\sum_{i=1}^{W} p_{i}^{q}}{q-1},
$$

where $W$ is the total number of configurations, $p_{i}$ are the associated probabilities, and $k_{B}$ is some suitable constant. Tsallis statistics is based on a special parameter $q$, which is adjusted to the phenomena represented by the theory. When $q \rightarrow 1$, the Boltzmann entropy is obtained. In [8], an inverse formalism was applied to find this limit. In this way an infinite number of entropies, and consequently of statistics, of Tsallis type were found. The necessity of deeper criteria to choose a formalism to represent physical systems was pointed out.

Naively describing, the method used in [8] consists in verifying that, for the special functional form of (1.1), separately integrating an infinite number of times the numerator and the denominator leads to the same result as differentiating a finite number of times (i.e, to apply the replica trick or, even simpler, L'Hôpital's rule). More precisely, one considers the Volterra operator $T$, as defined in Section 2, applies its iterates $T^{n}$ to the 
numerator and the denominator separately, and evaluates the limit of this expression as $n \rightarrow \infty$. Through this process, one achieves a limit which is independent of the parameter $q$. Here, we state and prove a theorem providing general conditions which allow one to apply this technique.

\section{The theorem}

For a real continuous function $f: \mathbb{R} \rightarrow \mathbb{R}$ and a point $x_{0} \in \mathbb{R}$, we denote by $T f(x)$ the Volterra operator

$$
T f(x)=\int_{x_{0}}^{x} f(t) d t
$$

For more results and properties of the Volterra operator and its iterates, see $[3,5-7,10$, 11].

To keep the notation simple, we do not write explicitly the dependence of the operator above on the point $x_{0}$. We use the usual notation $f^{(j)}(x)$ to denote the $j$ th derivative of $f(x)$. We begin with a simple lemma.

Lemma 2.1. Let $g: \mathbb{R} \rightarrow \mathbb{R}$ be a continuous function in an open neighborhood $U$ of $x_{0} \in \mathbb{R}$. If $f\left(x_{0}\right) \neq 0$, then there exists $\delta>0$ such that $V=\left[x_{0}-\delta, x_{0}+\delta\right] \subset U$ and $T^{n} f(x) \neq 0$ for all $n \in \mathbb{N}$ and all $x \in V \backslash\left\{x_{0}\right\}$.

Proof. Since $U$ is open and $f\left(x_{0}\right) \neq 0$, there exists $\delta>0$ such that $V=\left[x_{0}-\delta, x_{0}+\delta\right] \subset U$ and $f(x) \neq 0$, for all $x \in V$. We define $V_{1}:=\left(x_{0}, x_{0}+\delta\right]$ and $V_{2}:=\left[x_{0}-\delta, x_{0}\right)$. If $f(x)>0$ in $V=V_{1} \cup V_{2} \cup\left\{x_{0}\right\}$, we have

$$
\begin{aligned}
& T f(x)=\int_{x_{0}}^{x} f(t) d t>0, \quad \forall x \in V_{1}, \\
& T f(x)=\int_{x_{0}}^{x} f(t) d t<0, \quad \forall x \in V_{2} .
\end{aligned}
$$

If $f(x)<0$ in $V=V_{1} \cup V_{2} \cup\left\{x_{0}\right\}$, we have

$$
\begin{array}{ll}
T f(x)=\int_{x_{0}}^{x} f(t) d t<0, & \forall x \in V_{1}, \\
T f(x)=\int_{x_{0}}^{x} f(t) d t>0, & \forall x \in V_{2} .
\end{array}
$$

Therefore, in both cases, we have $T f(x) \neq 0$ for all $x \in V \backslash\left\{x_{0}\right\}$. Now suppose that for some $n \in \mathbb{N}$,

$$
T^{n} f(x) \neq 0,
$$

for all $x \in V \backslash\left\{x_{0}\right\}$. We analyze the signal of $T^{n+1} f(x)$ in the intervals $V_{1}$ and $V_{2}$ separately. For the interval $V_{1}$, we have that if $T^{n} f(x)>0$ in $V_{1}$, then

$$
T^{n+1} f(x)=T\left(T^{n} f\right)(x)=\int_{x_{0}}^{x} T^{n} f(t) d t>0, \quad \forall x \in V_{1},
$$


and if $T^{n} f(x)<0$ in $V_{1}$, then

$$
T^{n+1} f(x)=T\left(T^{n} f\right)(x)=\int_{x_{0}}^{x} T^{n} f(t) d t<0, \quad \forall x \in V_{1} .
$$

For the interval $V_{2}$, we have that if $T^{n} f(x)>0$ in $V_{2}$, then

$$
T^{n+1} f(x)=T\left(T^{n} f\right)(x)=\int_{x_{0}}^{x} T^{n} f(t) d t<0, \quad \forall x \in V_{2},
$$

and if $T^{n} f(x)<0$ in $V_{2}$, then

$$
T^{n+1} f(x)=T\left(T^{n} f\right)(x)=\int_{x_{0}}^{x} T^{n} f(t) d t>0, \quad \forall x \in V_{2} .
$$

Thus, we have $T^{n+1} f(x) \neq 0$ for all $x \in V_{1} \cup V_{2}=V \backslash\left\{x_{0}\right\}$. Therefore, it follows by induction that

$$
T^{n} f(x) \neq 0, \quad \forall x \in V \backslash\left\{x_{0}\right\}, \forall n \in \mathbb{N} .
$$

Corollary 2.2. Let $K \in \mathbb{N}$ and let $f: \mathbb{R} \rightarrow \mathbb{R}$ be a function of class $\mathscr{C}^{K+2}$ in an open neighborhood $U$ of $x_{0} \in \mathbb{R}$. Suppose $f^{(j)}\left(x_{0}\right)=0$, for $j=0,1, \ldots, K$, and $f^{(K+1)}\left(x_{0}\right) \neq 0$. Then, there exists $\delta>0$ such that $V=\left[x_{0}-\delta, x_{0}+\delta\right] \subset U$ and $T^{n} f(x) \neq 0$ for all $n \in \mathbb{N}$ and all $x \in V \backslash\left\{x_{0}\right\}$.

Proof. By Lemma 2.1, there exists $V=\left[x_{0}-\delta, x_{0}+\delta\right] \subset U, \delta>0$, such that

$$
T^{n} f^{(K+1)}(x) \neq 0
$$

for all $n \in \mathbb{N}$ and all $x \in V \backslash\left\{x_{0}\right\}$. Therefore, for all $m \in \mathbb{N}$, we have

$$
T^{m} f(x)=T^{m+K+1} f^{(K+1)}(x) \neq 0,
$$

for all $x \in V \backslash\left\{x_{0}\right\}$.

We now state and prove the main result.

Theorem 2.3. Let $f: \mathbb{R} \rightarrow \mathbb{R}, g: \mathbb{R} \rightarrow \mathbb{R}$ be functions of class $C^{K+2}$ in an open neighborhood $U$ of $x_{0} \in \mathbb{R}$. Suppose $f^{(j)}\left(x_{0}\right)=g^{(j)}\left(x_{0}\right)=0$, for $j=0,1, \ldots, K$, and $g^{(K+1)}\left(x_{0}\right) \neq 0$. Let $V=\left[x_{0}-\delta, x_{0}+\delta\right] \subset U, \delta>0$, be an interval where $T^{n} g(x) \neq 0$, for all $n \in \mathbb{N}$ and all $x \in V \backslash\left\{x_{0}\right\}$. Then,

$$
\lim _{n \rightarrow \infty} \frac{T^{n} f(x)}{T^{n} g(x)}=\frac{f^{(K+1)}\left(x_{0}\right)}{g^{(K+1)}\left(x_{0}\right)}
$$

uniformly in $V \backslash\left\{x_{0}\right\}$. 
4 Iterates of Volterra operators and indeterminate forms

Proof. The existence of the interval $V$ is assured by Corollary 2.2. Let $x \in V \backslash\left\{x_{0}\right\}$ and set either $I_{x}=\left[x_{0}, x\right]$ if $x>x_{0}$ or $I_{x}=\left[x, x_{0}\right]$ if $x<x_{0}$. Since $f$ and $g$ are of class $\varphi^{K+2}$ in $U \supset V \supset I_{x}$, and both have their first $K$ derivatives vanishing at $x_{0}$, there exist $c_{1}, c_{2} \in I_{x}$ such that

$$
\begin{aligned}
& f(x)=\frac{f^{(K+1)}\left(x_{0}\right)}{(K+1) !}\left|x-x_{0}\right|^{(K+1)}+\frac{f^{(K+2)}\left(c_{1}\right)}{(K+2) !}\left|x-x_{0}\right|^{(K+2)}, \\
& g(x)=\frac{g^{(K+1)}\left(x_{0}\right)}{(K+1) !}\left|x-x_{0}\right|^{(K+1)}+\frac{g^{(K+2)}\left(c_{2}\right)}{(K+2) !}\left|x-x_{0}\right|^{(K+2)} .
\end{aligned}
$$

Since $T^{n}$ is linear for all $n \in \mathbb{N}$, we have

$$
\begin{aligned}
& T^{n} f(x)=\frac{f^{(K+1)}\left(x_{0}\right)}{(K+1+n) !}\left|x-x_{0}\right|^{(K+1+n)}+\frac{f^{(K+2)}\left(c_{1}\right)}{(K+2+n) !}\left|x-x_{0}\right|^{(K+2+n)}, \\
& T^{n} g(x)=\frac{g^{(K+1)}\left(x_{0}\right)}{(K+1+n) !}\left|x-x_{0}\right|^{(K+1+n)}+\frac{g^{(K+2)}\left(c_{2}\right)}{(K+2+n) !}\left|x-x_{0}\right|^{(K+2+n)} .
\end{aligned}
$$

Therefore,

$$
\frac{T^{n} f(x)}{T^{n} g(x)}=\frac{f^{(K+1)}\left(x_{0}\right)+\left(f^{(K+2)}\left(c_{1}\right) /(K+2+n)\right)\left|x-x_{0}\right|}{g^{(K+1)}\left(x_{0}\right)+\left(g^{(K+2)}\left(c_{2}\right) /(K+2+n)\right)\left|x-x_{0}\right|} .
$$

Now, since $f^{(K+2)}$ and $g^{(K+2)}$ are both continuous in the compact set $V$, there exists $M>0$ such that $\left|f^{(K+2)}\left(c_{1}\right)\right| \leq M$ and $\left|g^{(K+2)}\left(c_{2}\right)\right| \leq M$. Moreover, we have $\left|x-x_{0}\right| \leq \delta$, since $x \in V \backslash\left\{x_{0}\right\}$. Thus,

$$
\begin{aligned}
& \left|\frac{f^{(K+2)}\left(c_{1}\right)}{K+2+n}\right|\left|x-x_{0}\right| \leq \frac{M \delta}{K+2+n} \\
& \left|\frac{g^{(K+2)}\left(c_{2}\right)}{K+2+n}\right|\left|x-x_{0}\right| \leq \frac{M \delta}{K+2+n} .
\end{aligned}
$$

These inequalities imply

$$
\lim _{n \rightarrow \infty} \frac{T^{n} f(x)}{T^{n} g(x)}=\frac{f^{(K+1)}\left(x_{0}\right)}{g^{(K+1)}\left(x_{0}\right)}
$$

Note that the limit is uniform for $x \in V \backslash\left\{x_{0}\right\}$ as desired, since inequalities (2.16) hold for all $x \in V \backslash\left\{x_{0}\right\}$ and all $c_{1}, c_{2} \in I_{x} \subset V$.

As an application of the result, one has that the family of entropies introduced in [8]

$$
{ }_{n} S_{q}=k_{B} \frac{\sum_{i=1}^{W} \sum_{k=0}^{n-1}\left(p_{i} / \ln ^{n-1-k} p_{i}\right)(q-1)^{k} / k !-\sum_{i=1}^{W}\left(p_{i}^{q} / \ln ^{n-1} p_{i}\right)}{(q-1)^{n} / n !}
$$


allows one to recover the Boltzmann-Gibbs entropy in the limit $n \rightarrow \infty$. Indeed, the expression in $(2.18)$ is obtained separately integrating $(n-1)$ times, with respect to $q$, the numerator and the denominator of the expression in (1.1) (see [8]). It is worth mentioning that at each iteration a new entropy is obtained with essentially the same properties of the original one. Therefore, through this process, one obtains an infinite set of entropies. Moreover, the method can be applied to a wide range of entropic forms, as, for example, Rényi's [4]

$$
S_{q}=\frac{\ln \left(\sum_{i=1}^{W} p_{i}^{q}\right)}{1-q}
$$

or Abe's [1]

$$
S_{q}=-k_{B} \frac{\sum_{i=1}^{W} p_{i}^{q}-\sum_{i=1}^{W} p_{i}^{1 / q}}{q-(1 / q)} .
$$

This opens the possibility of many applications and studies.

There are related methods to obtain a set of entropies. We mention here the works by Sharma and Mittal [9] and by van der Lubbe et al. [13] that have been also the subject of many recent works, and by Yamano [14], which includes the entropies of Tsallis and Rényi as particular cases.

\section{Conclusions}

The theorem proved allows one to solve, under quite general conditions, indeterminate forms through an infinite number of iterations of the Volterra operator. Through this process, an infinite number of indeterminate forms of the same type are obtained. Beyond this pure mathematical application, it is worth mentioning that for large $n$, oneparameter-dependent entropies ${ }_{n} S_{q}$ appear to be insensitive to the choice of $q$. This fact may help with the approximate evaluation of thermostatic properties of such systems. In view of the impact of generalized entropies in recent works, we believe that the result may find applications in physics and information theory.

\section{Acknowledgments}

The authors would like to thank the two anonymous referees for corrections in previous versions of the proofs, as well as for suggestions and criticisms that improved the result and the presentation of the paper. P. Braz e Silva also wishes to thank the International Centre for Theoretical Physics, Trieste, Italy, since this collaboration was made possible by a visit to the centre.

\section{References}

[1] S. Abe, A note on the q-deformation-theoretic aspect of the generalized entropies in nonextensive physics, Physics Letters. A 224 (1997), no. 6, 326-330.

[2] Z. Daróczy, Generalized information functions, Information and Computation 16 (1970), 36-51.

[3] S. P. Eveson, Asymptotic behaviour of iterates of Volterra operators on $L^{p}(0,1)$, Integral Equations and Operator Theory 53 (2005), no. 3, 331-341. 


\section{Iterates of Volterra operators and indeterminate forms}

[4] J. Karmeshu (ed.), Entropy Measures, Maximum Entropy Principle and Emerging Applications, Studies in Fuzziness and Soft Computing, vol. 119, Springer, Berlin, 2003.

[5] D. Kershaw, Operator norms of powers of the Volterra operator, Journal of Integral Equations and Applications 11 (1999), no. 3, 351-362.

[6] N. Lao and R. Whitley, Norms of powers of the Volterra operator, Integral Equations and Operator Theory 27 (1997), no. 4, 419-425.

[7] G. Little and J. B. Reade, Estimates for the norm of the nth indefinite integral, The Bulletin of the London Mathematical Society 30 (1998), no. 5, 539-542.

[8] A. R. R. Papa, On one-parameter-dependent generalizations of Boltzmann-Gibbs statistical mechanics, Journal of Physics. A. Mathematical and General 31 (1998), no. 23, 5271-5276.

[9] B. D. Sharma and D. P. Mittal, New nonadditive measures of entropy for discrete probability distributions, Journal of Mathematical Sciences 10 (1975), 28-40 (1976).

[10] B. Thorpe, The norm of powers of the indefinite integral operator on $(0,1)$, The Bulletin of the London Mathematical Society 30 (1998), no. 5, 543-548.

[11] F. G. Tricomi, Integral Equations, Pure and Applied Mathematics, vol. 5, Interscience, New York, 1957.

[12] C. Tsallis, Possible generalization of Boltzmann-Gibbs statistics, Journal of Statistical Physics 52 (1988), no. 1-2, 479-487.

[13] J. C. A. van der Lubbe, Y. Boxma, and D. E. Boekee, A generalized class of certainty and information measures, Information Sciences 32 (1984), no. 3, 187-215.

[14] T. Yamano, On a simple derivation of a family of nonextensive entropies from information content, Entropy 6 (2004), no. 4, 364-374.

Pablo Braz e Silva: Departamento de Matemática, Universidade Federal de Pernambuco, Av. Prof. Luiz Freire, s/n, Cidade Universitária, 50740-540 Recife, PE, Brazil E-mail address: pablo@dmat.ufpe.br

Andrés R. R. Papa: Observatório Nacional, MCT, Rua General José Cristino 77, São Cristovão, 20921-400 Rio de Janeiro, RJ, Brazil

Current address: Instituto de Física, Universidade do Estado do Rio de Janeiro, Rua São Francisco Xavier 524, Maracanã, 20550-900 Rio de Janeiro, RJ, Brazil E-mail address: papa@on.br 


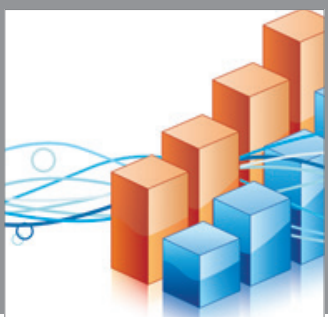

Advances in

Operations Research

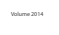

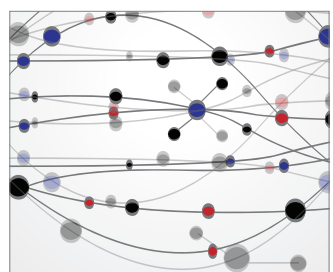

\section{The Scientific} World Journal
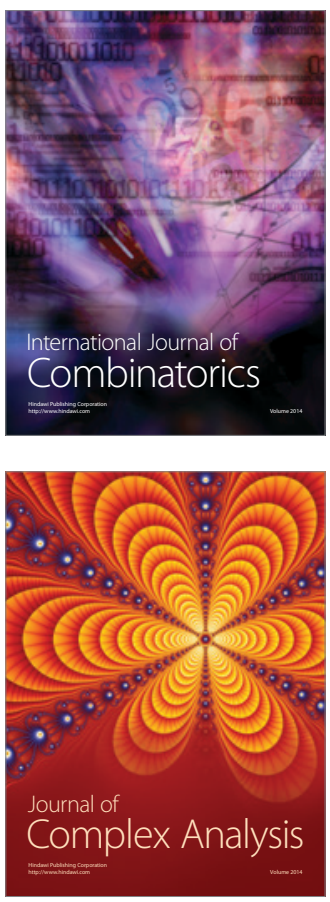

International Journal of

Mathematics and

Mathematical

Sciences
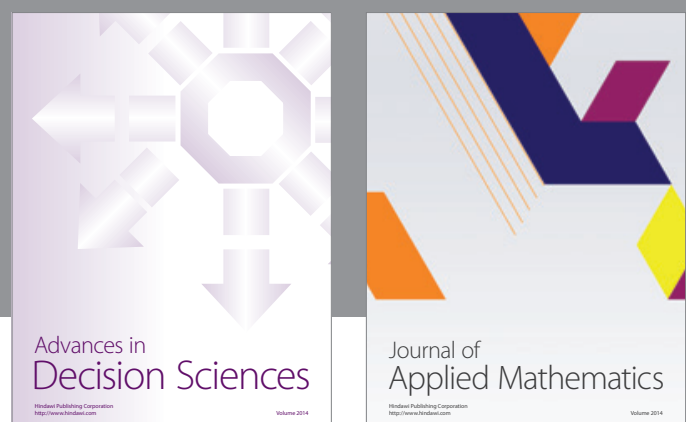

Journal of

Applied Mathematics
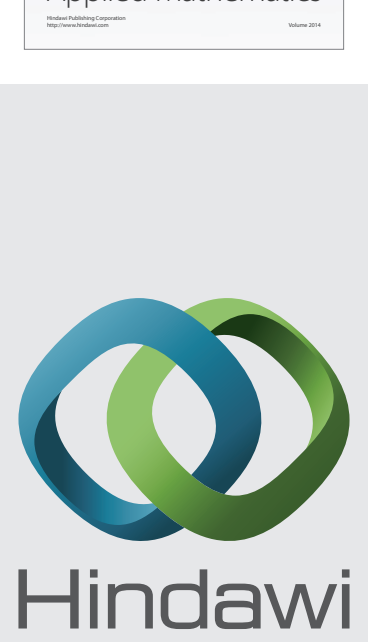

Submit your manuscripts at http://www.hindawi.com
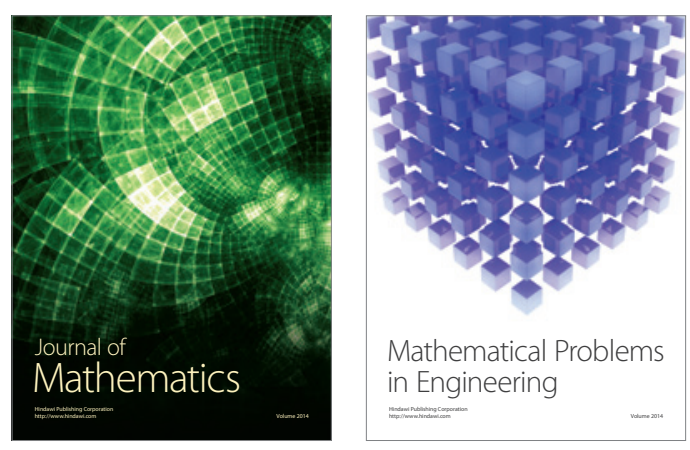

Mathematical Problems in Engineering
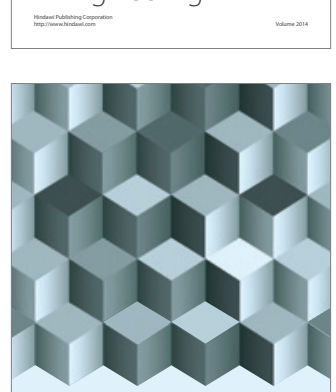

Journal of

Function Spaces
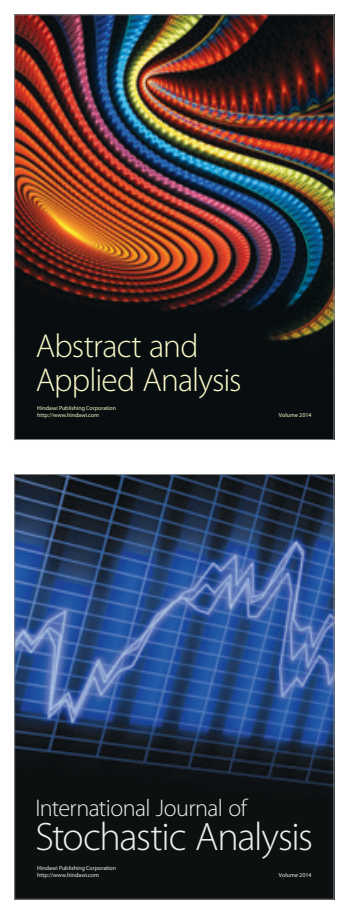

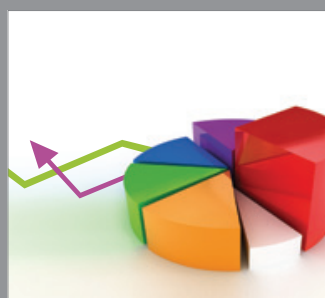

ournal of

Probability and Statistics

Promensencen
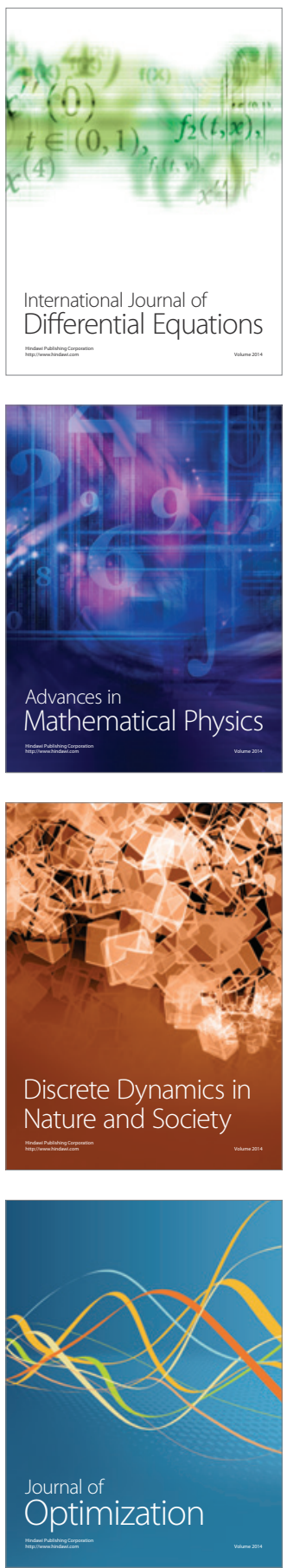